\title{
Magnetic helicity balance during a filament eruption that occurred in active region NOAA 9682
}

\author{
F. P. Zuccarello ${ }^{1,2}$, P. Romano ${ }^{2}$, F. Zuccarello ${ }^{3}$, and S. Poedts ${ }^{1}$ \\ 1 Centre for Plasma-Astrophysics, K.U.Leuven, Celestijnenlaan 200, 3001 Leuven, Belgium \\ e-mail: Francesco.Zuccarello@wis.kuleuven. be \\ 2 INAF - Osservatorio Astrofisico di Catania, via S. Sofia 78, 95123 Catania, Italy \\ 3 Dipartimento di Fisica e Astronomia - Universitá di Catania via S.Sofia 78, 95123 Catania, Italy
}

Received 11 February 2011 / Accepted 30 March 2011

\begin{abstract}
Context. Photospheric shear plasma flows in active regions may be responsible for the magnetic helicity injection in the solar corona not only during the energy storage process before a solar eruption, but also during and after the release of the free magnetic energy caused by the eruption. Indeed, after a filament eruption or expansion the magnetic torque imbalance can induce shear flows that can be responsible for yet another injection of magnetic helicity into the corona.

Aims. We investigated the magnetic helicity balance in an active region where a confined solar eruption occurred. This was done to verify a possible relationship between the filament expansion and the helicity transport at its footpoints. We aimed to verify if this variation in the helicity transport rate could be interpreted as a consequence of the magnetic torque imbalance caused by the tube expansion, as proposed by Chae et al. (2003, J. Kor. Astron. Soc., 36, 33).

Methods. We used $171 \AA$ TRACE data to measure some geometrical parameters of the new magnetic system produced by a filament eruption that occurred on 2001 November 1 in active region NOAA 9682. We used MDI full disk line-of-sight magnetogram data to measure the accumulation of magnetic helicity in the corona before and after the event.

Results. From the measured expansion factor in the magnetic arcade, visible at $171 \AA$ during the eruption, we estimated that the resulting torque imbalance at the photosphere ought to lead to the injection of negative helicity following the eruption. We compared this with measurements of the helicity injection using photospheric velocity and magnetogram data.

Conclusions. In contradiction to the expectations from the Chae et al. model, the helicity injection after the eruption was positive. We offer the alternative interpretation that the helicity injection resulted from torque of the opposite sign, generated as the filament lost its negative helicity through magnetic reconnection with its surroundings.
\end{abstract}

Key words. Sun: filaments, prominences - Sun: activity - magnetic fields

\section{Introduction}

Recently, many authors have studied the magnetic helicity variations in active regions where solar eruptions took place (Moon et al. 2002; Nindos et al. 2003; Romano et al. 2005; Zhang et al. 2009; Smyrli et al. 2010). Indeed, the magnetic helicity provides a measure of the global complexity of the magnetic field in an active region, i.e., a measure of the writhe and twist of the magnetic flux tubes, whose interaction favors processes of magnetic reconnection and the consequent release of free magnetic energy.

However, ambiguous results have been obtained mainly due to the difficulties in determining this physical quantity in the corona using the boundary conditions provided by the photospheric magnetic field measurements. Therefore, many authors preferred to consider the magnetic helicity variations instead of the estimation of the helicity itself. In particular, Pariat et al. (2005) have developed a new method of determining the magnetic helicity flux using a time series of line-of-sight magnetogram data.

To understand the above mentioned relationship between the solar eruptions and the magnetic helicity variations, Smyrli et al. (2010) applied this method to MDI/SOHO line-of-sight magnetogram data to calculate the magnetic helicity trend in 10 active regions that gave rise to halo coronal mass ejections (CMEs). The results obtained from the sample of analyzed events indicate that the changes in magnetic helicity flux following the CMEs could be attributed to a process of restoring a torque balance between the subphotospheric and the coronal domain of the flux tubes.

This process has been described by Longcope \& Welsch (2000) for the emergence of new magnetic flux into the corona. In their model, only a fraction of the current carried by the emerging twisted flux tube passes into the corona. As a consequence, a torsional Alfvén wave is launched downward along the flux tube at the instant of emergence. As the flux tube continues to emerge, the helicity of the coronal field increases owing to rotation of the foot points. Similarly, in solar eruptions, the magnetic helicity injected by shear flows is interpreted by Chae et al. (2003) as the process of transferring magnetic helicity from the interior to the corona to restore the torque balance that was lost with the sudden expansion of the flux tube involved in the event. In particular, Chae et al. (2003) derived a simple relationship between the expansion parameter of the coronal segment of a twisted flux tube that is rooted deeply below the photosphere and the amount of helicity transferred via shear flows. Therefore, if we consider a CME or an eruptive filament as an example of this coronal expansion, the sudden magnetic shear increase observed in major flares (Wang 1994) and the sudden variations of magnetic helicity change rate (Moon et al. 2002) may be attributed to 
this process. In this paper, we consider a well observed filament eruption that was already studied by Romano et al. (2011, hereafter Paper I). This eruption occurred on 2001 November 1 in the active region NOAA 9682, was observed by TRACE at $171 \AA$ and showed the expansion of a highly sheared arcade during the main phase of the filament eruption. Therefore, it constitutes a good target for the measure of the coronal expansion parameter proposed by Chae et al. (2003) and for its comparison with the magnetic helicity variations measured after the eruption from the MDI line-of sight photospheric magnetograms of the active region. We organized our paper as follows. Section 2 gives a description of the data analysis and the results. The discussion is presented in Sect. 3 and the conclusions are drawn in Sect. 4.

\section{Data analysis}

In the active region NOAA 9682, on 2001 November 1 between 11:00 UT and 12:30 UT a filament eruption was observed by TRACE at $171 \AA$ with a field of view of $768 \times 768$ pixels, a spatial resolution of 1 arcsec and a temporal resolution of $42 \mathrm{~s}$ (see the TRACE movie at http://trace.lmsal.com/ POD/movies/T171_011101_1130eruption.mov). A comparison between the $\mathrm{H}_{\alpha}$ images (high-resolution BBSO images and INAF-OACt images) and the line-of-sight magnetogram taken by MDI on 2001 November 1 at 11:11 UT enabled us to locate the filament foot points before the eruption (see Paper I, Fig. 2).

Before the eruption, the filament, which seems to be characterized by a negative chirality, can be identified with a thin and dark EUV channel (Fig. 1(a)a), whose westward foot point anchored in the most western sunspot of positive polarity and the eastward foot point anchored in the proximity of the active region center. At around 11:30 UT, the filament starts to show some brightenings in the TRACE images and a highly sheared arcade appears during the main phase of the eruption. During the event the western foot point seems to stay roughly in the same initial location, while the eastern one definitely shows a northeastward drift motion (compare Figs. 1(a)a and b). The filament stops to be visible in the TRACE images at around 12:30 UT.

According to Chae et al. (2003), during the expansion of a twisted flux tube the magnetic torque imbalance that is generated at the photosphere should transfer in the coronal part of the tube an amount of helicity $\Delta H$ given by

$\Delta H=(\gamma-1) H_{\mathrm{i}}$,

where

$\gamma=\frac{L_{\exp }}{l_{\mathrm{i}}} \frac{\mathrm{d} R_{\exp }}{R_{\exp }} \frac{r_{\mathrm{i}}}{\mathrm{d} r_{\mathrm{i}}}$

is the expansion factor with $H_{\mathrm{i}}$ corresponding to the initial helicity, $l_{\mathrm{i}}, r_{\mathrm{i}}$ and $\mathrm{d} r_{\mathrm{i}}$ corresponding to the length, radius, and radial thickness of an annulus of the initial flux tube, respectively, while $L_{\exp }, R_{\exp }$ and $\mathrm{d} R_{\exp }$ are corresponding to the length, radius, and radial thickness of an annulus of the expanded flux tube. When the expansion is uniform along the radius, $\gamma$ is given only by the ratio between the length of the flux tube when expanded and the length of the flux tube before the expansion.

Assuming that the filament involved in the eruption is a flux tube characterized by a helically twisted magnetic field, we considered two different TRACE images to measure this expansion factor: one when the filament is still in its initial equilibrium and one when it reaches the maximum of its expansion. Figure 1(a)a shows the image taken at 10:56 UT, before the expansion started. The black line identifies the EUV counterpart of the filament,

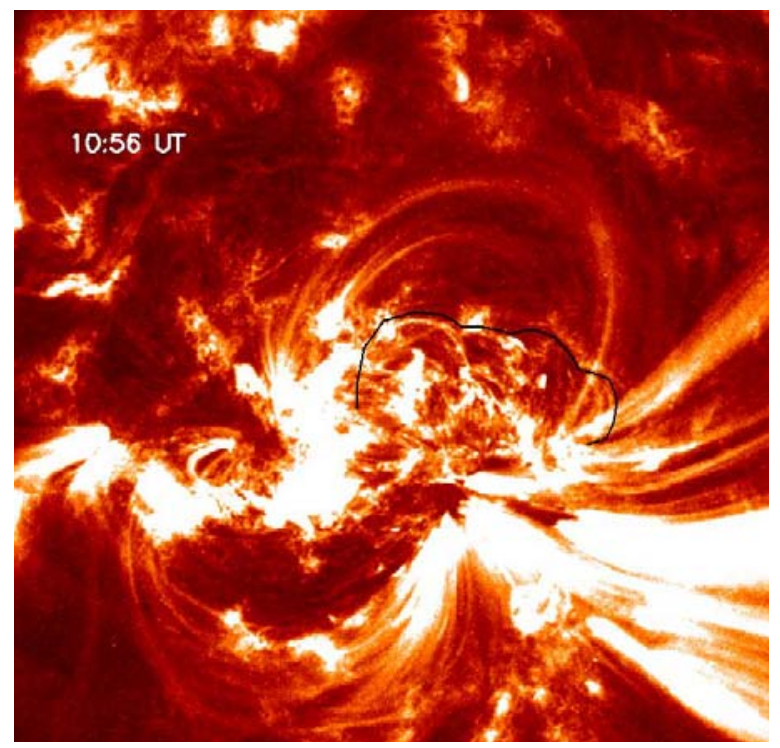

(a)

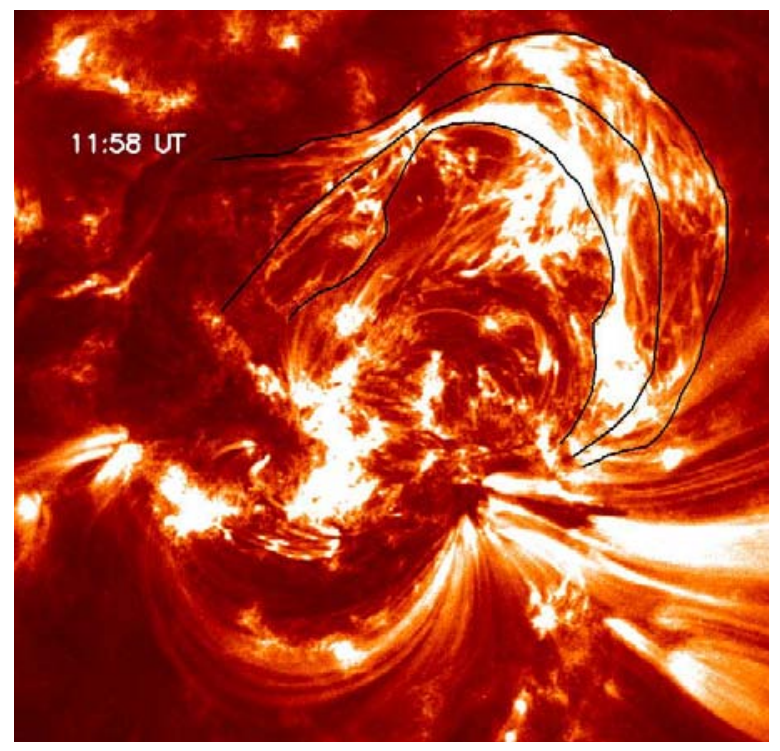

(b)

Fig. 1. TRACE images taken at different times. a) At 10:56 UT the EUV filament channel is outlined by the black line. b) At 11:58 UT the filament is expanded. Solid lines outline the filament boundaries and axis. The field of view is $\sim 280 \times 280 \mathrm{Mm}^{2}$. North is at the top and west to the right.

whose length is $l_{\mathrm{i}} \simeq 1.55 \times 10^{5} \mathrm{~km}$. Figure 1 (a)b shows the image taken at 11:58 UT, near the peak of the arcade expansion. As a consequence of the north-eastward drift motion, the identification of the eastern foot point is less straightforward. To measure the length of the axis, we first identified the outer and the inner boundaries of the filament, then we outlined the axis of the filament. The length of the axis in the expanded configuration is $L_{\text {exp }} \simeq 3.1 \times 10^{5} \mathrm{~km}$. We used an IDL procedure (Contarino et al. 2006) based on a model developed by Loughhead et al. (1983) to estimate the influence of the projection effects on the length measurements. We found that the discrepancy between the projected length and the reconstructed length is less than $10 \%$. With these measured values of $l_{\mathrm{i}}$ and $L_{\mathrm{exp}}$, assuming an expansion uniform along the radius, we obtain an expansion factor $\gamma=2.02$. 


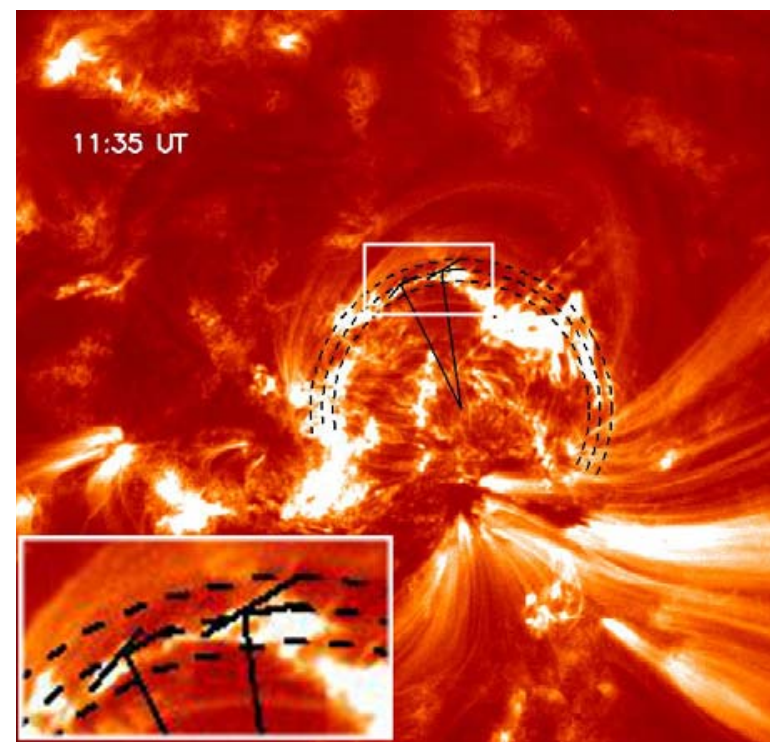

Fig. 2. At 11:35 UT the filament started to expand. The dashed lines outline the filament edges and axis, while the solid lines indicate the radius of the circumference. The two pitch angles (see text) are identified in the bottom left zoom. The field of view is $\sim 280 \times 280 \mathrm{Mm}^{2}$. North is at the top and west to the right.

Approximating the filament as a cylindrical curved flux tube, its initial helicity can be calculated as $H_{\mathrm{i}}=N_{\mathrm{i}} \Phi^{2}$, where $N_{\mathrm{i}}$ is the number of turns of the helix and $\Phi$ is the magnetic flux. For an uniformly twisted cylindrical flux tube the number of turns is given by (Vrsnak 1990)

$N_{\mathrm{i}}=l_{0} \frac{\tan \theta}{2 \pi r_{0}}$,

where $\theta$ is the angle that the field line forms with the axis of the tube, $r_{0}$ is the radius of the flux tube section and $l_{0}=R_{0} \times(\pi+$ $2 \arccos \left(d / R_{0}\right)$ is the length of the coronal part of the axis of the tube (where $2 d$ is the separation of the footpoints and $R_{0}$ is the radius of the torus).

To compute the number $N_{\mathrm{i}}$, we used the first available TRACE image in which some signatures of the helical thread of the filament were observable. Figure 2 shows the image at 11:35 UT when the filament just started to erupt. We fitted the filament within two concentric circumferences, corresponding to the outer and inner edges of the flux tube, and we identified the axis of the filament as being the circumference with a radius equal to $\left(R_{\text {ext }}+R_{\text {int }}\right) / 2$. We located the bright signatures that resemble the helical structure of the magnetic field and then calculated the angle between these bright features and the axis of the tube. On the circumference resembling the flux tube axis we identified the two foot points and calculated the half-foot-point separation $d \simeq 4.78 \times 10^{4} \mathrm{~km}$. The radius of the flux tube section is $r_{0} \simeq 4 \times 10^{3} \mathrm{~km}$, the length of the axis is $l_{0} \simeq 2.1 \times 10^{5} \mathrm{~km}$ and the radius of the circumference is $r_{0} \simeq 5.4 \times 10^{4} \mathrm{~km}$. With this cylindrical flux tube approximation the number of turns is $N_{\mathrm{i}} \simeq 3.78$.

Finally, in the magnetogram we identified the western foot point of the filament (cf. Paper I) and calculated the magnetic flux in that area of the magnetogram. The obtained magnetic flux is $\Phi=1.81 \times 10^{21} \mathrm{Mx}$. Therefore, if we assume a dextral filament, $H_{\mathrm{i}}=N_{\mathrm{i}} \Phi^{2} \simeq-1.23 \times 10^{43} \mathrm{Mx}^{2}$. From Eq. (1) with $\gamma=$ $L_{\text {Exp }} / l_{0}=1.47$, we obtain $\Delta H \simeq-5.8 \times 10^{42} \mathrm{Mx}^{2}$. However, this estimate is made after the filament started to expand. Therefore, the helicity variation due to the expansion is underestimated.

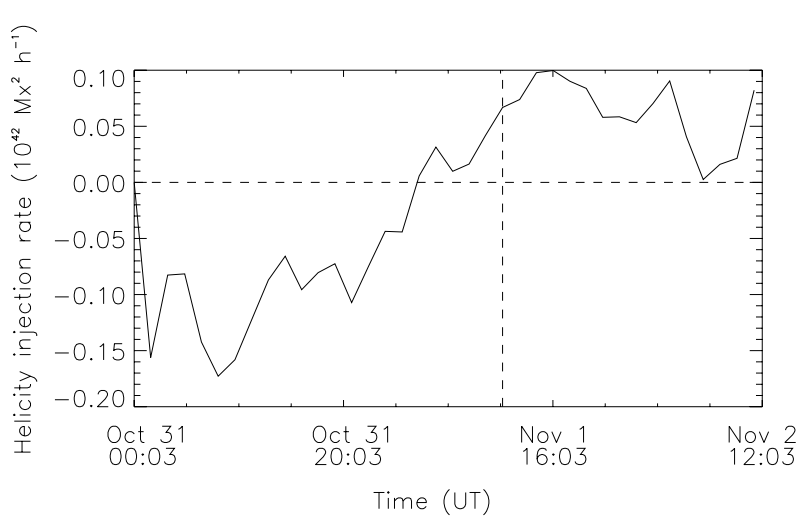

(a)

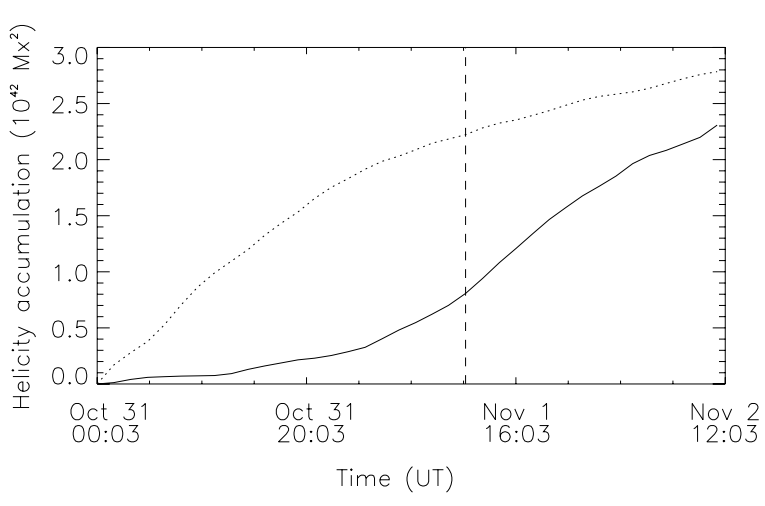

(b)

Fig. 3. Helicity accumulation around the western footpoint of the filament. The dashed vertical line indicates the time of the eruption. a) shows the helicity injection rate; b) shows the positive helicity accumulation (solid line) and the negative helicity accumulation (dotted line).

A more suitable helicity estimation can be found considering the initial length of the filament, i.e. $l_{i}$, instead of $l_{0}$. With this assumption we obtain $\gamma=2.02$ and $\Delta H \simeq-1.25 \times 10^{43} \mathrm{Mx}^{2}$. This is the magnetic helicity that should be transferred in the coronal volume as a consequence of the magnetic torque imbalance that is generated by the axial expansion of the filament.

To verify this hypothesis, we analyzed the full-disk line-ofsight magnetograms taken by MDI/SOHO at $6767.8 \AA$ with a spatial resolution of 3.96 arcsec and a temporal resolution of $96 \mathrm{~min}$. We considered magnetograms taken from 00:03 UT on 2001 October 31 to 12:51 UT on 2001 November 2, i.e., up to one day after the filament eruption. We were not able to follow the MDI evolution any longer because the active region reached a heliographic angle greater than 35 degrees, which introduced significant projection errors. We corrected all the magnetogram data for the angle between the magnetic field direction and the observer's line-of-sight. We considered subfields of $396 \times 396 \operatorname{arcsec}(200 \times 200$ pixels $)$ centered on NOAA 9682 and aligned all subfields by applying a standard differential rotation rate (Howard et al. 1990) with a sampling of 1 arcsec, i.e., implementing a subpixelization.

We calculated the helicity accumulation as the time integral of the helicity injection rate (Pariat et al. 2005):

$\frac{\mathrm{d} H}{\mathrm{~d} t}=-\frac{1}{2 \pi} \int_{S} \int_{S^{\prime}} \frac{\mathrm{d} \Theta(\boldsymbol{r})}{\mathrm{d} t} B_{n} B_{n}^{\prime} \mathrm{d} S \mathrm{~d} S^{\prime}$, 
where $\frac{\mathrm{d} \Theta(\boldsymbol{r})}{\mathrm{d} t}=r^{-2}\left(\boldsymbol{r} \times\left(\boldsymbol{u}-\boldsymbol{u}^{\prime}\right)\right)_{n}$ is the rotation rate of the two points located at $\boldsymbol{x}$ and $\boldsymbol{x}^{\prime}$, having a normal component of the magnetic field of $B_{n}$ and $B_{n}^{\prime}$, respectively. From the sequence of co-aligned MDI magnetograms, we determined the photospheric velocity maps using the differential affine velocity estimator (DAVE) (Schuck 2005). The DAVE method applies an affine velocity profile to a windowed aperture and is consistent with the magnetic induction equation. We used a full-widthat-half maximum of the apodization windows of 19.80 arcsec. Because the western foot point seems to stay almost in the same position during the total duration of the event, we calculated the helicity accumulation in an area of $20 \times 17$ pixels around this foot point, where the total magnetic flux is constant during the analyzed period. Figure 3 a shows the helicity injection rate through this foot point. During the first $30 \mathrm{~h}$ of observation, mainly negative helicity is injected into the solar corona, the major contribution coming from the filament foot point. Before the eruption, a change in the trend is observed and positive helicity starts to be injected. This injection becomes even more evident after the eruption. Figure $3 \mathrm{~b}$ shows the helicity accumulation separated into the positive and negative contributions. During the first $30 \mathrm{~h}$ the negative helicity injected is almost 4 times higher than the positive one. Before the eruption the trend of negative helicity injection decreases, while the positive helicity injection increases. This new trend seems to continue till the end of our observations.

\section{Discussion}

In the previous section, we deduced the magnetic helicity that should be transferred to the corona as a consequence of the magnetic torque imbalance. This helicity should be injected into the coronal volume on a time scale $\tau=l / v_{\text {a }}$ (Longcope \& Welsch 2000). Assuming a subphotospheric Alfvén velocity $v_{\mathrm{A}} \simeq 10^{5} \mathrm{~cm} \mathrm{~s}^{-1}$, it should take 3.5 days to transfer the amount $\Delta H$ of helicity. However, as already stressed by Chae et al. (2003), if the flux tube evolves on a shorter time scale than the Alfvén crossing time, it could never reach the torque balance, and the helicity pumped into the corona would be less than $\Delta H$.

As a consequence of this mechanism, negative magnetic helicity should be transferred through the photosphere after the filament eruption. This helicity transfer leads to transient shear flows that can be detected with the method described in the previous section, eventually enabling us to measure the helicity accumulation after the event. As can be deduced from Fig. 3 and as discussed in Paper I, at the foot points of the filament, persistent paths of negative helicity density are observed before the eruption eventually increases the negative helicity of the filament. However, after the eruption these negative helicity density paths became less persistent at least in the western foot point, which resulted in a decrease in the slope of the negative helicity accumulation.

Therefore, these observations do not seem to confirm what was expected by the torque imbalance model. Indeed, if we assume that the amount $\Delta H$ of negative helicity would have been transferred in about 3.5 days, this would have lead to a helicity accumulation of about $3.5 \times 10^{42} \mathrm{Mx}^{2} \mathrm{day}^{-1}$, making the slope in Fig. 3b (dotted line) even steeper. On the other hand, the positive helicity accumulation shows a change in the slope some hours before the eruption. Moreover, around the western foot point area, an amount of positive helicity about 4 times higher than during the previous $35 \mathrm{~h}$ is injected in the $24 \mathrm{~h}$ after the eruption.
This increase in the positive magnetic helicity can be a consequence of the torque imbalance. As already discussed in Paper I, the filament has a left-handed chirality, but belongs to an active region that shows a dominant positive magnetic helicity. In Paper I we suggested that the filament eruption may be caused by magnetic reconnection between two magnetic field systems characterized by opposite signs of magnetic helicity. Because the eruption failed, i.e., no coronal mass ejection was associated with this event, the total helicity of the system did not change.

Owing to the complexity of the event, the simple helicityconserving expansion described in Chae et al. (2003) is no longer valid. When the expanding dextral filament interacts with its surroundings, it transfers negative helicity through the reconnection to the flux system that is characterized by a positive chirality, which becomes visible during the eruption and eventually decreases its magnetic helicity. The existence of this new magnetic system that formed during the reconnection process seems to be confirmed in the TRACE images. Indeed, during the event, crossings of the type II, which indicate a right-handed flux tube (Chae 2000), are seen in the western side of the arcade, while crossings of type III, which lead to a negative helicity, are seen in the eastern part. Moreover, the TRACE movie shows torsional motions typical of a right-handed helix that unwinds, like a positive helicity magnetic flux tube that looses its helicity.

We therefore propose the following scenario. The active region could be resembled by a right-handed flux tube. The lefthanded filament, which undergoes the expansion, looses its integrity after interacting with the surrounding (right-handed) flux tube, eventually violating the helicity-conserving expansion hypothesis of Chae et al. (2003). The net effect of this interaction is the transfer of an amount $H_{\mathrm{i}}$ of (negative) magnetic helicity to the positive flux tube, which unwinds, resulting in a torque imbalance between its coronal part and its subphotospheric part. In other words, we propose that the shear flows that provided the positive helicity injection are not a consequence of the torque imbalance caused by the coronal expansion of the dextral filament (that should have lead to a negative helicity injection), but are caused by the torque imbalance that is generated as a consequence of the unwinding of the right-handed flux tube, which in this context better resembles the isolated flux tube described in Chae et al. (2003).

If we assume this scenario, the positive helicity lost by the system is $H_{\mathrm{i}}=N_{\mathrm{i}} \Phi^{2}=1.23 \times 10^{43} \mathrm{Mx}^{2}$. In the $24 \mathrm{~h}$ after the eruption, an amount of $\Delta H \simeq 1.6 \times 10^{42} \mathrm{Mx}^{2}$ of positive magnetic helicity is injected through photospheric motions into the area around the western foot point. This indicates that it would take about 3.7 days to transfer the helicity $H_{\mathrm{i}}$, to eventually reestablish the torque balance. This time is compatible with the time $\tau$ previously discussed.

\section{Conclusions}

Based on the idea proposed by Chae et al. (2003), we calculated the helicity accumulation that should be expected after a filament eruption as a consequence of the magnetic torque imbalance that is generated at the photospheric interface of the magnetic flux tube. The analysis of $\mathrm{H}_{\alpha}$ images and the results of Paper I strongly indicate the dextral nature of the filament, so that, according to the model, a negative helicity transfer should be expected after the eruption occurrence. We measured the helicity accumulation at the filament western foot point to verify this hypothesis.

We found that, in contradiction with what was expected, positive helicity was mainly injected into the corona after the 
filament erupted. Before the event (about 10-15 h) a change in the helicity injection rate is observed. Probably the filament is activated during this phase, resulting in a process of slow reconnection that starts to transfer negative helicity from the filament to the surrounding magnetic field. After the eruption, the helicity injection rate seems to stabilize around positive values, resulting in the further injection of positive magnetic helicity into the corona. This positive helicity injection could be caused by the strong magnetic torque imbalance that is generated as a consequence of the interaction between the dextral filament and the right-handed flux system, more than the filament expansion itself.

This event involved systems with different chiralities, maybe resulting in helicity transfer between the different systems. We argued that the helicity injection after the event could be ascribed to the magnetic torque imbalance even though, owing to the complexity of the event, we could not identify a sharp change in the helicity injection trend after the event.

In the near future we plan to investigate other active regions to verify if it is possible to identify changes in the helicity injection trends that could unambiguously be ascribed to the magnetic torque imbalance that is generated after the eruption.
Acknowledgements. These results were obtained in the framework of the projects GOA/2009-009 (K.U. Leuven), G.0304.07 (FWO-Vlaanderen), and C 90347 (ESA Prodex 9). Financial support by the European Commission through the SOLAIRE Network (MTRN-CT-2006-035484) is gratefully acknowledged.

\section{References}

Chae, J. 2000, ApJ, 540, L115

Chae, J., Moon, Y., Rust, D. M., Wang, H., \& Goode, P. R. 2003, J. Kor. Astron. Soc., 36, 33

Contarino, L., Romano, P., \& Zuccarello, F. 2006, Mem. Soc. Astron. Ital. Suppl., 9, 94

Howard, R. F., Harvey, J. W., \& Forgach, S. 1990, Sol. Phys., 130, 295

Longcope, D. W., \& Welsch, B. T. 2000, ApJ, 545, 1089

Loughhead, R. E., Wang, J., \& Blows, G. 1983, ApJ, 274, 883

Moon, Y., Chae, J., Wang, H., Choe, G. S., \& Park, Y. D. 2002, ApJ, 580, 528

Nindos, A., Zhang, J., \& Zhang, H. 2003, ApJ, 594, 1033

Pariat, E., Démoulin, P., \& Berger, M. A. 2005, A\&A, 439, 1191

Romano, P., Contarino, L., \& Zuccarello, F. 2005, A\&A, 433, 683

Romano, P., Pariat, E., Sicari, M., \& Zuccarello, F. 2011, A\&A, 525, A13

Schuck, P. W. 2005, ApJ, 632, L53

Smyrli, A., Zuccarello, F., Romano, P., et al. 2010, A\&A, 521, A56

Vrsnak, B. 1990, Sol. Phys., 129, 295

Wang, J. 1994, Sol. Phys., 155, 285

Zhang, Y., Tan, B., \& Yan, Y. 2009, ApJ, 704, 1622 